\title{
Gallium Ga 68-NODAGA-E(c[RGDyK])2
}

National Cancer Institute

\section{Source}

National Cancer Institute. Gallium Ga 68-NODAGA-E(C[RGDyK7)2. NCI Thesaurus. Code C132993.

A radiopharmaceutical agent composed of a dimeric cyclic arg inine-glycine-aspartic acid (RGD)-based peptide (c[RGDyK]) and labeled, via the chelating agent 1,4,7triazacyclononane,1-glutaric acid-4,7-acetic acid (NODAGA), with gallium Ga 68, with potential alphaVbeta3 (aVb3) integ rin imaging activity upon positron emission topography (PET) or single photon emission computed tomography (SPECT). After intravenous administration of gallium Ga 68-NODAGA-E(c[RGDyK])2, the cyclic RGD moiety targets and binds to $\mathrm{aVb} 3$ integ rin which is expressed on the cell membrane of tumor cells and tumor-resident endothelial cells. Upon imaging, aVb3 integrin-expressing cells can be visualized and expression levels can be quantified. This can be used to assess response to anti-angiogenic treatment. aVb3 integ rin is overexpressed on certain tumor cells and tumor-resident endothelial cells, while minimally or not expressed on healthy, normal cells; aVb3 integrin plays a key role in angiogenesis, tumor proliferation and survival. 